Pacific Journal of Mathematics

ON GENERALIZATIONS OF SYLOW TOWER GROUPS 


\section{ON GENERALIZATIONS OF SYLOW TOWER GROUPS}

\section{Abiabdollah FAtTAhi}

In this paper two different generalizations of Sylow tower groups are studied. In Chapter I the notion of a $k$-tower group is introduced and a bound on the nilpotence length (Fitting height) of an arbitrary finite solvable group is found. In the same chapter a different proof to a theorem of Baer is given; and the list of all minimal-not-Sylow tower groups is obtained.

Further results are obtained on a different generalization of Sylow tower groups, called Generalized Sylow Tower Groups (GSTG) by J. Derr. It is shown that the class of all GSTG's of a fixed complexion form a saturated formation, and a structure theorem for all such groups is given.

\section{NOTATIONS}

The following notations will be used throughont this paper:

$N \triangleleft G$

$N$ Char $G$

$N \cdot \triangleleft G$

$M<G$

$M<\cdot G$

$Z(G)$

$|G|_{p}$

$\pi(G)$

$\phi(G)$

$[H] K$

$F(G)$

$C(H)=C_{G}(H)$

$N(H)=N_{G}(H)$

$P \in \operatorname{Syl}_{p}(G)$

$P$ is a $S_{p}$-subgroup of $G$

Core $(H)=\operatorname{Core}_{G}(H)$

$l(G)$

$l_{p}(G)$

$d(G)$

$c(P)$

$p^{*}$

$O_{p}(G)$
$N$ is a normal subgroup of $G$

$N$ is a characteristic subgroup of $G$

$N$ is a minimal normal subgroup of $G$

$M$ is a proper subgroup of $G$

$M$ is a maximal subgroup of $G$

the center of $G$

$p$-part of the order of $G, p$ a prime

set of all prime divisors of $|G|$

the Frattini subgroup of $G=$ the intersec-

tion of all maximal subgroups of $G$ semi-direct product of $H$ by $K$ the Fitting subgroup of $G=$ the maximal normal nilpotent subgroup of $G$ the centralizer of $H$ in $G$ the normalizer of $H$ in $G$

$P$ is a Sylow $p$-subgroup of $G$

$P \in \operatorname{Syl}_{p}(G)$

the largest normal subgroup of $G$ contained in $H=\bigcap_{g \in G} H^{g}$

the nilpotence length (Fitting height) of $G$ $p$-length of $G$

minimal number of generators of $G$ nilpotence class of the $p$-group $P$ some nonnegative power of prime $p$ largest normal $p$-subgroup of $G$ 
$O_{p}(G)$

$O_{p^{\prime} p}(G)$

$Z^{\infty}(G)$

$G \sim H$

$G \sim{ }_{r} H$

STG

GSTG

$U>\triangleleft G$

$\Sigma_{n}$

$A_{n}$ largest normal subgroup of $G$ of order relatively prime to $p$

the inverse image in $G$ of $O_{p}\left(G / O_{p}(G)\right)$

the hypercenter of $G=$ the last term in the upper central series of $G$

the wreath product of $G$ by $H$

the regular wreath product of $G$ by $H$

Sylow tower group

generalized Sylow tower group

$U$ is an abnormal subgroup of $G$

symmetric group on $n$ letters

alternating group on $n$ letters

All the groups we consider are assumed to be finite.

\section{CHAPTER I}

\section{$k$-Tower and Sylow Tower Groups}

1. k-tower groups. The concept of a $k$-tower group provides an alternative way of looking at finite solvable groups and enables us to obtain some results about such groups by generalizing some of the properties of Sylow tower groups. We start with the definition of a $k$-tower group:

Definition. Let $G$ be a finite group with $|G|=\prod_{i=1}^{n} p_{i}^{r_{i}}$ and $k$ be a positive integer. We say that $G$ has a $k$-tower if there are subgroups $H_{i} \unlhd G, i=0,1,2, \cdots, k n$ such that

$$
1=H_{0} \unlhd H_{1} \unlhd H_{2} \unlhd \cdots \unlhd H_{k n}=G,
$$

where each $\left|H_{i+1} / H_{i}\right|=p_{j}^{s}, s_{j} \geqq 0(j=1,2, \cdots, n)$ and the number of nontrivial $p_{j}$-factors is at most $k$ for all $j$ and is precisely $k$ for at least one $j$.

Observations. The followings are immediate consequences of this definition:

(a) $G$ has a 1-tower $\Leftrightarrow G$ has a Sylow tower.

This is just the definition of a Sylow tower group.

(b) $G$ has a k-tower $\Rightarrow$ every subgroup and every homomorphic image of $G$ has a $k^{\prime}$-tower for some $k^{\prime} \leqq k$.

If $H \leqq G$, the tower obtained from the $k$-tower of $G$ by intersecting each entry with $H$ is a $k^{\prime}$-tower for $H$ and since some of these intersections may be the identity, so $k^{\prime} \leqq k$. The $k^{\prime}$-tower for the homomorphic image is obtained by taking the homomorphic image of each 
entry in the $k$-tower of $G$.

(c) $G$ has a k-tower for some $k \Rightarrow G$ is solvable.

This can be seen by induction on $|G|$. Let $H$ be the first nonidentity entry in the $k$-tower of $G$. Then $G / H$ has a $k^{\prime}$-tower and since $|G| H \mid<$ $|G|$ so $G / H$ is solvable by induction. But $H$ is a $p$-group so $G$ is solvable.

(d) $G$ is solvable $\Rightarrow G$ has a k-tower for some $k$. Any chief series of $G$ can be thought of as a $k$-tower for $G$.

(e) If $G_{1}$ has a $k_{1}$-tower and $G_{2}$ has a $k_{2}$-tower, then $G_{1} \times G_{2}$ has a k-tower for some $k \leqq k_{1}+k_{2}$.

If $1=H_{0} \unlhd H_{1} \unlhd \cdots \unlhd H_{k_{1} n_{1}}=G_{1}$ and $1=k_{0} \unlhd k_{1} \unlhd \cdots \unlhd k_{k_{2} n_{2}}=G_{2}$ are the $k_{1}$-tower of $G_{1}$ and $k_{2}$-tower of $G_{2}$ respectively, then

$$
1=H_{0} \unlhd H_{1} \unlhd \cdots \unlhd H_{k_{1} n_{1}}=G_{1} \unlhd G_{1} \times K_{1} \unlhd \cdots \unlhd G_{1} \times G_{2}
$$

is a $k$-tower for $G_{1} \times G_{2}$.

(f) If we further assume that in part $(e), \pi\left(G_{1}\right) \cap \pi\left(G_{2}\right)=\phi$, then $G_{1} \times G_{2}$ has a k-tower for some $k \leqq \operatorname{Max}\left\{k_{1}, k_{2}\right\}$.

This is clear.

Definition. Suppose $G$ has a $k$-tower $1=H_{0} \unlhd H_{1} \unlhd \cdots \unlhd H_{k n}=$ $G$. We define the type of $G$ to be the ordered kn-tuple

$$
\left(p_{1}, p_{2}, \cdots, p_{i}, \cdots, p_{k n}\right),
$$

consisting of the primes that appear in the factors of this tower. Note that some of these factors may be trivial in which case we can put any prime in its corresponding place in the $k n$-tuple.

If $G$ has a $k$-tower of a given type, then any subgroup and any homomorphic image of $G$ has a $k^{\prime}$-tower of the same type for some $k^{\prime} \leqq k$ (note that in the $k^{\prime} n^{\prime}$-tuple we can always insert enough 1 's in the right places to make it into a $k n$-tuple). Since the direct product of two $k$-tower groups of the same type is clearly again a $k$-tower of that type, we get:

COROLlaRY 1.1. The class of all groups having a $k^{\prime}$-tower of a given type for some $k^{\prime} \leqq k$ is a formation.

The next result shows that this formation is saturated:

THEOREM 1.2. If $G / \phi(G)$ has a k-tower of a given type, then so does G. Moreover, if for some prime $p_{0}$, the number of nontrivial $p_{0}$-factors in the $k$-tower of $G / \phi(G)$ is equal to $k^{\prime}<k$, the same holds for $G$.

Proof. We will prove the whole statement of the theorem by 
induction on $|G|$. Let $K / \phi(G)$ be the first nonidentity entry in the $k$-tower of $G / \phi(G)$. Then $K \triangleleft G$ and $K / \phi(G)$ is a $p$-group, for some $p$. Let $P \in \operatorname{Syl}_{p}(K)$. By Frattini argument, $G=K N_{G}(P)$. Since $P \phi(G) / \phi(G)$ is a $S_{p}$-subgroup of $K / \phi(G)$ and $K / \phi(G)$ is a $p$-group, so we get $P \phi(G)=K$. Hence,

$$
G=K N_{G}(P)=N_{G}(P) K=N_{G}(P) P \phi(G)=N_{G}(P) \dot{\phi}(G) \text {. }
$$

Therefore, $G=N_{G}(P)$, and we get $P \triangleleft G$.

Now since $\phi(G / P) \geqq \phi(G) P / P=K / P$, we have that $G / P / \phi(G / P)$ is a homomorphic image of $G / P / K / P \approx G / K$. But $G / K$ has a $k$-tower and the number of $p$-factors in that tower is $\leqq k-1$. So $G / P / \phi(G / P)$ has a $k_{1}$-tower for some $k_{1} \leqq k$ and the number of nonidentity $p$-factors is $\leqq k_{1}-1 \leqq k-1$. So by induction $G / P$ has a $k_{1}$-tower and number of $p$-factors in that tower is $\leqq k_{1}-1$. Since $P$ is $p$-group and $P \triangleleft$ $G$, we get that $G$ has a $k_{1}$-tower, $k_{1} \leqq k$. On the other hand, $G / \phi(G)$ is a homomorphic image of $G$, so $k \leqq k_{1}$. Therefore, $k_{1}=k$, i.e., $G$ has a $k$-tower. If for some prime $p_{0} \neq p$, the number of $p_{0}$-factors of $G / \varphi(G)$ is equal to $k^{\prime}<k$, the same holds for $G / K$ and, consequently, the same thing will have to hold for $G$.

If $G$ is a Sylow tower group, then $p$-length of $G$ is $\leqq 1$ for all primes $p$. This result generalizes to $k$-tower groups as follows:

THEOREM 1.3. If $G$ has a k-tower, then $l_{p}(G) \leqq k$, for all $p$.

Proof. The $k$-tower of $G$ is a normal series each of whose factors is either a $p$-group or a $p^{\prime}$-group, the number of $p$-factors not exceeding $k$. This being true for each prime $p$, we conclude that $l_{p}(G) \leqq k$, for all $p$.

CoROLlary 1.4. Suppose $G \leqq H=H_{1} H_{2} \cdots H_{m}$, where $H_{i} \triangleleft H$ and each $H_{i}$ has a $k$-tower. Then $G$ is solvable and $l_{p}(G) \leqq k$, for all $p$.

Proof. Each $H_{i}$ is solvable, and $l_{p}\left(H_{i}\right) \leqq k$, for all $p$ and all $i=1,2, \cdots, m$ by Theorem 1.3. So $G$ is solvable and since

$$
l_{p}(H)=\operatorname{Max}\left\{l_{p}\left(H_{1}\right), l_{p}\left(H_{2}\right), \cdots, l_{p}\left(H_{m}\right)\right\},
$$

so $l_{p}(G) \leqq l_{p}(H) \leqq k$.

Our next goal is to prove the converse of 1.4, i.e.;

THEOREM 1.5. Suppose $G$ is a solvable group with $l_{p}(G) \leqq k$ for all $p$. Then $G \lesssim H=H_{1} H_{2} \cdots H_{m}$, where each $H_{i}$ has a $k$-tower, and 
furthermore, if $l_{p_{0}}(G) \leqq k^{\prime}<k$ for some $p_{0}$, then the number of nontrivial $p_{0}$-factors in the $k$-tower of each $H_{i}$ is $\leqq k^{\prime}$.

Note. This generalizes a theorem of J. L. Alperin about Sylow tower groups [1] to $k$-tower groups.

Proof of 1.5. We proceed by induction on $|G|$. We can assume that $G$ has a unique minimal normal subgroup. Because if $N_{1}$ and $N_{2}$ are two distinct minimal normal subgroups of $G$, then $N_{1} \cap N_{2}=$ 1. But $G / N_{1} \cap N_{2} \lesssim G / N_{1} \times G / N_{2}$ and $G / N_{i}, i=1,2$ satisfies the hypothesis of the theorem, so by induction, $G / N_{i}, i=1,2$ has the desired form and therefore so does $G$.

Let $N$ be the unique minimal normal subgroup of $G$. Since $G$ is solvable, $|N|=p^{r}$, for some prime $p$ and some positive integer $r$. So $K=O_{p}(G)>1$ and $O_{p^{\prime}}(G)=1$. Consequently, $O_{p^{\prime} p}(G)=O_{p}(G)=K$, and

$$
l_{p}(G / K)=l_{p}\left(G / O_{p^{\prime}}(G)\right)=l_{p}(G)-1 \leqq k-1 .
$$

Clearly, $l_{q}(G / K) \leqq l_{q}(G) \leqq k$, for all primes $q \neq p$. Since $|G / K|<|G|$, by induction we get that

$$
G / K \lesssim L=L_{1} L_{2} \cdots L_{m},
$$

where $L_{i} \triangleleft L$, each $L_{i}$ has a $k$-tower, and since $l_{p}(G / K) \leqq k-1$, by second part of the theorem the number of nonidentity $p$-factors in the $k$-tower of each $L_{i}$ is $\leqq k-1$.

By [7, pp. 98, 99] we have:

$$
G \lesssim K \sim_{r} G / K{ }_{r} K \sim_{r} L .
$$

But the wreath product $k \sim_{r} L$ can be expressed as a semi-direct product, namely $H=K \sim_{r} L \approx K^{*} L^{*}$, where $K^{*}=K \times K \times \cdots \times K$ $\left(|L|\right.$ times) and $L^{*} \approx L$. So $K^{*}$ is a $p$-group and $K^{*} \triangleleft H$. Furthermore, $H=K^{*} L=K^{*}\left(L_{1} L_{2} \cdots L_{m}\right)=H_{1} H_{2} \cdots H_{m}$, where $H_{i} \approx K^{*} L_{i} \triangleleft H$, $i=1,2, \cdots, m$. show:

So we have shown that $G \leqq H=H_{1} H_{2} \cdots H_{m}$, and it remains to

(i) each $H_{i}$ has a $k$-tower;

(ii) if $l_{p_{0}}(G) \leqq k^{\prime}<k$ for some $p_{0}$, then the number of nonidentity $p_{0}$-factors in the $k$-tower of each $H_{i}$ is $\leqq k^{\prime}$.

(i): If $1=L_{i}^{0} \unlhd L_{i}^{1} \unlhd \cdots \unlhd L_{i}^{k n}=L_{i}$ is a $k$-tower for $L_{i}$, then $1 \triangleleft K^{*} \unlhd K^{*} L_{i}^{1} \unlhd \cdots \unlhd K^{*} L_{i} \approx H_{i}$ is a $k$-tower for $H_{i}$. Note since the number of nonidentity $p$-factors in the $k$-tower of $L_{i}$ is $\leqq k-1$ and since $K^{*}$ is a $p$-group, so the number of nonidentity $p$-factors in the tower of $H_{i}$ is still $\leqq k$.

(ii): If $l_{p_{0}}(G) \leqq k^{\prime}<k$ for some $p_{0}$, then $l_{p_{0}}(G / K) \leqq k^{\prime}$ (and if 
$p_{0}=p$, then $\left.l_{p_{0}}(G / K) \leqq k^{\prime}-1\right)$ and hence by induction each $L_{i}$ has $\leqq k^{\prime}$ nonidentity $p_{0}$-factors in its $k$-tower $\left(\leqq k^{\prime}-1\right.$, if $\left.p_{0}=p\right)$. Thus, the same holds for $H_{i} \approx K^{*} L_{i}$.

REMARK. It is clear from the proof that $H$ can be choosen so that $\pi(H)=\pi(G)$.

As a consequence of Theorem 1.5 we get a bound for the nilpotence length of a finite solvable group in terms of the minimal number of generators or the nilpotence class of its Sylow subgroups. We need the following lemma first:

Lemma 1.6. Suppose $H$ and $K$ are two normal subgroups of $G$ with $l(H) \leqq k_{1}$ and $l(K) \leqq k_{2}$. Let $k=\operatorname{Max}\left\{k_{1}, k_{2}\right\}$. Then $l(H K) \leqq k$. (Here $l(H)$ denotes the nilpotence length (Fitting height) of $H$. )

Proof. By induction on $k$. If $k=1$, then $H$ and $K$ are nilpotent normal subgroups of $G$ and Fitting's theorem yields that $H K$ is also nilpotent. Therefore, we may assume $k \geqq 2$. Since $F(H)$ char $H$ and $H \triangleleft G$ so $F(H) \triangleleft G$. Similarly, $F(K) \triangleleft G$. Therefore, $F(H) F(K) \triangleleft$ G. We write:

$$
\begin{aligned}
H K / F(H) F(K) & =H F(H) K F(K) / F(H) F(K) \\
& =(H F(K) / F(H) F(K))(K F(H) / F(H) F(K)) .
\end{aligned}
$$

Since $F(H) \leqq F(H) F(K), H F(K) / F(H) F(K)$ is a homomorphic image of $H F(K) / F(H)$. But $H F(K) / F(H)=(H / F(H))(F(K) F(H) / F(H))$. Here, $l(H / F(H))=l(H)-1 \leqq k-1$, and $l(F(H) F(K) / F(H))=1$, as $F(K)$ is nilpotent. So by induction $l(H F(K) / F(H)) \leqq k-1$. Similarly, $l(K F(H) / F(H) F(K)) \leqq k-1$.

Now, $\left({ }^{*}\right)$ and induction yield that $l(H K / F(H) F(K)) \leqq k-1$. But $F(H) F(K) \leqq F(H K)$, so $l(H K / F(H K)) \leqq k-1$ and, therefore, $l(H K) \leqq$ $k$.

If $G$ is a group, we denote by $G_{p}$ a Sylow $p$-subgroup of $G$. Let $d\left(G_{p}\right)$ and $c\left(G_{p}\right)$ denote the minimal number of generators and the nilpotence class of $G_{p}$ respectively.

Theorem 1.7. Let $G$ be any finite solvable group. Set $k_{p}=$ $\min \left\{d\left(G_{p}\right), c\left(G_{p}\right)\right\}$, for each prime $p$ dividing $|G|$. If $k=\sum_{p|| G \mid} k_{p}$, then $l(G) \leqq k$.

Proof. By Hall-Higman's result $l_{p}(G) \leqq k_{p}$ for each $p$. So if we set $k_{1}=\operatorname{Max}_{p|| G \mid}\left\{k_{p}\right\}$, then $l_{p}(G) \leqq k_{1}$ for all $p$. So by Theorem 1.5, $G \lesssim H_{1} H_{2} \cdots H_{m}$, where each $H_{i}$ has a $k_{1}$-tower and $\pi\left(H_{i}\right)=\pi(G)$. By the second part of Theorem 1.5 there are at most $k_{p}$ nonidentity $p$ - 
factors in the $k_{1}$-tower of each $H_{i}$, for all $p$. This means that $l\left(H_{i}\right) \leqq$ $\sum_{p|| G \mid} k_{p}=k$. Now a repeated use of Lemma 1.6 yields that $l(H) \leqq$ $k$. This in turn gives us $l(G) \leqq k$.

REMARK. If $k^{\prime}=\max _{p|| G \mid}\left\{k_{p}\right\}$, and $n=$ the number of distinct prime divisors of $|G|$, then it is clear from Theorem 1.7 that $l(G) \leqq$ $k^{\prime} n$. In particular, if $G$ is an A-group (i.e., has Abelian Sylow subgroups), then $l(G) \leqq$ number of distinct prime divisors of $|G|$.

2. Sylow tower groups. In this section we give an alternative proof for Theorem 2.1 below. Two different proofs are already given to this theorem by J. Thompson [9] and R. Bear [2]. A third proof, using a result of Gaschütz, was suggested to me by Professor J. Alperin and the work is presented in this section. Later I found out that a graph-theoretical proof of this theorem was given by T. O. Hawkes [6].

THEOREM 2.1. If every 2-generator subgroup of a finite group $G$ has a Sylow tower, then so does $G$.

It will be sufficient to prove the following

THEOREM 2.2. If every proper subgroup and every proper homomorphic image of a finite solvable group $G$ has a Sylow tower but $G$ does not, then $d(G)=2$.

Definition. A group satisfying the hypothesis of Theorem 2.2 will be called a critical (STG-Critical) group.

We now show that Theorem 2.1 follows from Theorem 2.2.

Suppose Theorem 2.1 is false. Choose $G$ minimal with the property that every 2-generator subgroup of $G$ has a Sylow tower but $G$ does not. Then every proper subgroup of $G$ has a Sylow tower by minimality of $G$. If we set $G^{*}=G / \phi(G)$, then clearly every proper subgroup of $G^{*}$ has a Sylow tower. Also if $1<N^{*} \triangleleft G^{*}$, then since

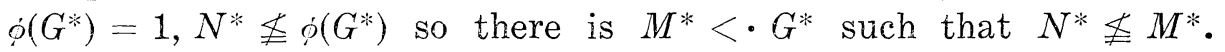
Then

$$
G^{*} / N^{*}=M^{*} N^{*} / N^{*} \approx M^{*} / M^{*} \cap N^{*} .
$$

So $G^{*} / N^{*}$ being isomorphic to a homomorphic image of a proper subgroup of $G^{*}$, has a Sylow tower. This shows that every proper homomorphic image of $G^{*}$ also has a Sylow tower, i.e., $G^{*}$ is a criti- 
cal group. If $G^{*}$ is not solvable, then it must be simple. Indeed it must be minimal simple, as all its proper subgroups being Sylow tower groups are solvable. But by Thompson's classification of all minimal simple groups we know that all such groups are generated by two elements. So in this case we get $d\left(G^{*}\right)=2$. If $G^{*}$ is solvable, then by Theorem 2.2 we know that $d\left(G^{*}\right)=2$. So in any case we get that

$$
d(G)=d\left(G^{*}\right)=2 .
$$

But this means that $G$ has a Sylow tower since every 2-generator subgroup of $G$ does by hypothesis. This contradicts the choice of $G$ and proves our claim.

We therefore begin to prove Theorem 2.2. For the remainder of this section $G$ will denote a solvable critical group. $G$ has the following additional properties:

(1) $G$ does not have any normal Hall subgroup. If $H$ were a nontrivial normal Hall subgroup of $G$, then $H$ and $G / H$ would have Sylow towers and this implies that $G$ has a Sylow tower.

(2) $\phi(G)=1$.

Otherwise $G / \phi(G)$ has a Sylow tower and then by Theorem 1.2 so does $G$.

(3) $Z(G)=1$.

If $Z(G)>1$, let $P \leqq Z(G),|P|=p$. Since $\phi(G)=1, P$ has a complement $S$ in $G$ and since $P$ is central, $G=S \times P$. But $S$ has a Sylow tower, therefore, so does $G$.

(4) $F(G)=A_{1} \times A_{2} \times \cdots \times A_{k}$, where $A_{i} \cdot \triangleleft G$ and $\left|A_{i}\right|=p_{i}^{a_{i}}$. This follows from the fact that in any finite group $G, F(G) / \phi(G)$ is the direct product of Abelian minimal normal subgroups of $G / \phi(G)$ [7, p. 279]. Here $\phi(G)=1$ and $G$ is solvable.

(5) $l_{p}(G) \leqq 1$, for all $p$.

If $l_{p_{0}}(G)>1$ for some prime $p_{0}$, then by Powell's theorem [3] there exists a 2-generator subgroup $H$ of $G$ such that $l_{p_{0}}(H)>1$. But $l_{p}(H) \leqq 1$ for all $p$ as $H$ has a Sylow tower.

(6) Lemma (Bear [2]). For each $p \| G: G^{\prime} \mid$ there is a normal subgroup $N$ of $G$ with $|G: N|=p$. Furthermore, $N$ has a unique $S_{p}$-subgroup $M$ which is at the same time a minimal normal subgroup of $G$.

Proof (Baer [2]). Since $G / G^{\prime}$ is Abelian there is $N / G^{\prime} \triangleleft G / G^{\prime}$ with $\left|G / G^{\prime}: N / G^{\prime}\right|=p$. So $|G: N|=p$. Since $N$ is a proper subgroup of $G$, it must have a Sylow tower and, therefore, possesses a normal $S_{q}$-subgroup for some prime $q$. If $q \neq p$, then $G$ also has a normal $S_{q}$-subgroup violating (1). So $p=q$ and $N$ has a (unique) normal $S_{p^{-}}$ 
suogroup $M$. Clearly $M \triangleleft G$.

Choose $M_{0} \cdot \triangleleft G$ such that $M_{0} \leqq M$. We want to show $M_{0}=M$.

Since $\phi(G)=1$, there is $S<\cdot G$ with $S M_{0}=G$ and $S \cap M_{0}=1$. By Dedekind identity we get

$$
M=M_{0}(M \cap S) \text {. }
$$

$S$ has a Sylow tower and so has a normal $q$-complement $T$ for some $q$. If $q \neq p$, then $M_{0} T$ is a normal $q$-complement for $G$, violating (1). So $p=q$.

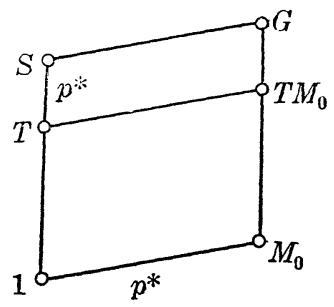

FIGURE 1

Now, $M \cap S \triangleleft S$ and $M \cap S$ is normalized by $M_{0}$ as $M_{0} \leqq Z(M)$ (by minimality of $M_{0}$ ). So $M \cap S \triangleleft G$. So

$$
G / C(M \cap S) \lesssim \operatorname{Aut}(M \cap S)
$$

Since $T$ and $M \cap S$ are normal subgroups of $S$ of coprime order, we have

$$
T \leqq C_{S}(M \cap S) \leqq C(M \cap S)
$$

So $G / C(M \cap S)$ is a $p$-group. But then (*) shows that $G$ induces a $p$-group of automorphism in the normal $p$-subgroup $M \cap S$. So it fixes some element $1 \neq z \in M \cap S$, i.e., $z \in Z(G)$. This is a contradiction unless $M \cap S=1$ in which case we get $M_{0}=M$.

This shows that if $|G|=\prod_{i=1}^{n} p_{i}^{r_{i}}$ and if $p_{i} \| G: G^{\prime} \mid$, then $G$ has a minimal normal subgroup $A_{i}$ with $\left|A_{i}\right|=p_{i}^{r_{i}^{-1}}$. Since $G$ has no normal Sylow subgroup this would be the only minimal normal subgroup of $G$ of order a power of $p_{i}$. In particular, this shows that in (4) each $A_{i}$ is a $S_{p_{i}}$-subgroup of $F(G)$.

Let $\left\{P_{1}, P_{2}, \cdots, P_{n}\right\}$ be a Sylow basis for $G$ and let $D$ be the corresponding system-normalizer. Suppose $p \| G: G^{\prime} \mid$. So $p=p_{i}$ for some $i=1,2, \cdots, n$. Let $P$ be the corresponding Sylow subgroup in the above basis. Set $K=O_{p^{\prime}}(G)$.

$P K / K$ is a $S_{p}$-subgroup of $G / K$. So by (5), $P K / K \triangleleft G / K$. Therefore, by Schur-Zassenhaus theorem there is $R / K \leqq G / K$ with:

$$
(P K / K)(R / K)=G / K \text { and } P K \cap R=K \text {. }
$$


So we get

$$
G=P R \quad \text { and } \quad P K \cap R=K .
$$

Let $M$ be the minimal normal subgroup of $G$ obtained in (6). We have the following diagram:

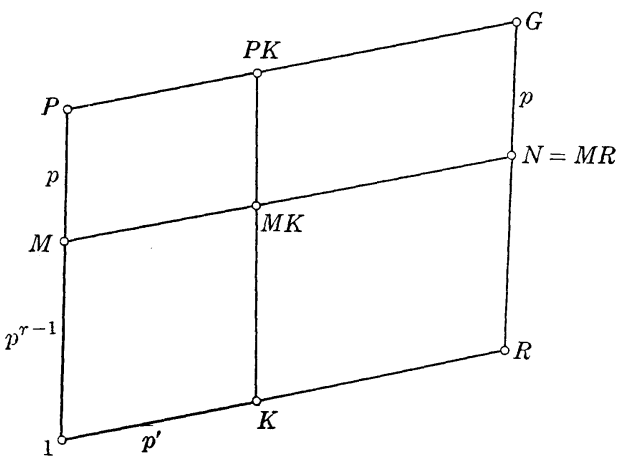

FIGURE 2

It is clear from this diagram that $M \approx M K / K$ and $P K / M K$ are the only $p$-chief factors of $G$ in the chief series obtained by refining the series $1 \triangleleft K \triangleleft M K \triangleleft P K \triangleleft G$.

(7) $P K / M K$ and $M K / K$ are both complemented chief factors. The complement to $P K / M K$ is $N / M K$ as it can be seen from the diagram. Since $\phi(G)=1$ and $M \cdot \triangleleft G, M$ has a complement $H$ in $G$, i.e., there is $H<G$ with

$$
G=H M \text { and } H \cap M=1 .
$$

Now it is clear that $H / K$ is a complement to $M K / K$ (note that since $H$ is a complement to $M$, it must contain a Hall $p^{\prime}$-subgroup of $G$ and so $H \geqq K)$.

(8) $P K / M K$ is a central and $M K / K$ is an eccentric chief factor of $G$.

$P K / M K$ and $N / M K$ are normal subgroups of $G / M K$ of coprime orders, so they centralize each other. But $P K / M K$ has order $p$ so $P K / M K \leqq$ $Z(G / M K)$. This shows that $P K / M K$ is central.

If $M K / K \leqq Z(G / K)$, then $[M K, G] \leqq K$. So $[M, G] \leqq K$. But then $[M, G] \leqq K \cap M=1$, i.e., $M \leqq Z(G)=1$, a contradiction. So $M K / K$ is eccentric.

(9) $F(G) \cap D=1$.

Since $F(G)=A_{1} \times A_{2} \times \cdots \times A_{k}$ and $A_{i}$ are all eccentric chief factors of $G, D$ avoids each $A_{i}$ and so it avoids every factor in:

$$
1<A_{1}<A_{1} A_{2}<\cdots<A_{1} A_{2} \cdots A_{k}=F(G) \text {. }
$$

It follows that $D$ avoids $F(G)$, i.e., $F(G) \cap D=1$. 
(10) $\pi(F(G)) \subseteq \pi\left(G / G^{\prime}\right)$.

Let $p \| F(G) \mid$ and let $A$ be a minimal normal subgroup of $G$ of $p$ power order. Then $G / A$ has a Sylow tower and so it has normal $q$-complement for some prime $q$. If $q \neq p$, then $G$ also has a normal $q$-complement violating (1). So $q=p$ and if $L / A$ is the normal $p$-complement of $G / A$, then $|G: L|=p$, i.e., $G^{\prime} \leqq L$. So $p \| G: G^{\prime} \mid$.

(11) $\pi(F(G)) \subseteq \pi(D)$.

This is clear from the diagram $\left(^{*}\right)$ as $G / N$ is a central chief factor and so $D$ covers it, i.e., $p \| D \mid$.

Our final goal is to find all chief factors of $G$ and determine whether they are complemented, central, or eccentric. We have this information about those chief factors whose orders are divisible by prime divisors of $\left|G: G^{\prime}\right|$. We will show that $\pi\left(G / G^{\prime}\right)=\pi(G)$. To establish this we use some of the ideas from Thompson's proof of Theorem 2.2.

We choose notation so that $\pi(F(G))=\left\{p_{1}, p_{2}, \cdots, p_{k}\right\}, k \leqq n$. For each $p_{i}, i=1,2, \cdots, k$ choose $x_{i} \in D$ with $\left|x_{i}\right|=p_{i}$. This can be done by (11). Let $X=\left\langle x_{1}, x_{2}, \cdots, x_{k}\right\rangle$. Since $X \leqq D, X$ is nilpotent. Since $F(G) X \leqq G$ we have:

$$
p_{i}^{r_{i}}=|G|_{p_{i}} \geqq|F(G) X|_{p_{i}} .
$$

So

$$
p_{i}^{r_{i}} \geqq \frac{|F(G)|_{p_{i}}|X|_{p_{i}}}{|F(G) \cap X|_{p_{i}}}=|F(G)|_{p_{i}}|X|_{p_{i}}=p_{i}^{r_{i}-1}|X|_{p_{i}} .
$$

Since $p_{i}|| X \mid$, we get that $|X|_{p_{i}}=p_{i}, i=1,2, \cdots, k$. So $X$ is cyclic of order $p_{1} p_{2} \cdots p_{k}$.

(12) $x_{i} \in C\left(A_{i}\right), i=1,2, \cdots, k$.

Let $p=p_{i}$. Note that since $M K / K$ has a complement in $G / K$, it has a complement $S / K$ in $P K / K$. But $M K / K \leqq Z(P K / K)$, as $M K / K \cdot \triangleleft$ $G / K$ and $P K / K$ is a normal $S_{p}$-subgroup of $G / K$. So $P K / K$ has a central subgroup with a cyclic quotient and is therefore Abelian. It is actually elementary Abelian. Since $P K / K \approx P$, this means that all $S_{p_{i}}$-subgroups of $G$ are elementary Abelian for $i=1,2, \cdots, k$. Since $x_{i}$ is a $p_{i}$-element so $x_{i} \in P_{i}$ for some $S_{p_{i}}$-subgroup $P_{i}$ of $G$. But $A_{i} \leqq P_{i}$. So $P_{i}=A_{i} \times\left\langle x_{i}\right\rangle$, as $x_{i} \notin A_{i}$ and $P_{i}$ and $A_{i}$ are elementary Abelian. This proves (12).

(13) $\quad G=F(G) X$.

Let $H=F(G) X$. Clearly $F(H) \geqq F(G)$. If actually $F(H)>F(G)$, then $F(H)$ must contain some $x_{i}$. Since $F(H)$ is nilpotent and $F(G) \leqq F(H)$ so $x_{i}$ centralizes all $p_{i}^{\prime}$-elements of $F(G)$. But by (12) $x_{i}$ also centralizes all $p_{i}$-elements of $F(G)$. So $\left.x_{i} \in C(F G)\right) \leqq F(G)$. But this cannot happen as $F(G) \cap D=1$. So $F(G)=F(H)$. If $K$ 
is a normal $p_{i}$-subgroup of $H$ containing $x_{i}$, then $K \leqq F(H)=F(G)$ which is not possible, as $x_{i} \notin F(G)$. So $H$ has no normal $S_{p_{i}}$-subgroup. Thus, $H$ has no Sylow tower. So we must have $H=G$.

Now it is clear from (13) that $\pi(G)=\pi(F(G))$ and this combined with (10) and (11) gives:

(14) $\pi(G)=\pi(F(G))=\pi(D)=\pi\left(G / G^{\prime}\right)$.

This means that the diagram $\left(^{*}\right)$ exists for all prime divisors of $|G|$ and so we know all the chief factors of $G$. By using the following formula of Gaschütz [5] we can compute the number of different 2generator systems of $G$ :

$$
\begin{aligned}
\phi_{G}(s)= & \prod_{i=1}^{h}\left|\mathscr{E}_{i}\right|^{\beta_{i} s} \prod_{k=1}^{h}\left(\left|\mathscr{E}_{k}\right|^{s}-\left|\mathscr{E}_{k}\right|^{\xi_{k}}\right) \\
& \times\left(\left|\mathscr{E}_{k}\right|^{s}-\left|\mathscr{E}_{k}\right|^{\xi_{k}}\left|\boldsymbol{E}_{k}\right|\right) \cdots\left(\left|\mathscr{E}_{k}\right|^{s}-\left|\mathscr{E}_{k}\right|^{\xi_{k}}\left|E_{k}\right|^{\alpha_{k}-1}\right) .
\end{aligned}
$$

Here, $\mathscr{E}_{1}, \mathscr{E}_{2}, \cdots, \mathscr{C}_{h}$ are the representatives of $G$-isomorphism classes of simple $G$-groups. In each isomorphism class $\mathscr{E}_{i}$ there are $\alpha_{i}$ complemented and $\beta_{i}$ noncomplemented members. $\xi_{i}$ is defined by

$$
\xi_{i}=\left\{\begin{array}{l}
0 \text { if } G \text { acts trivially on members of } \mathscr{E}_{i} \\
1 \text { otherwise }
\end{array}\right.
$$

and $E_{i}=$ endomorphism ring of $\mathscr{E}_{i}$. Finally $\phi_{G}(s)$ is the number of different $s$-generator system for $G$. If we can show that $\phi_{G}(2)>0$, we are done.

Let $\mathscr{E}_{1}, \mathscr{C}_{2}, \cdots, \mathscr{E}_{n}$ be the eccentric chief factors of order $p_{i}^{r_{i}-1}$ and $\mathscr{E}_{n+1}, \mathscr{E}_{n+2}, \cdots, \mathscr{E}_{2 n}$ be the central ones of order $p_{i}$. We have:

$$
\begin{aligned}
s & =2, h=2 n \\
\alpha_{i} & =1, i=1,2, \cdots, 2 n \\
\beta_{i} & =0, i=1,2, \cdots, 2 n \\
\left|\mathscr{E}_{i}\right| & =p_{i}^{r_{i}-1}, i=1,2, \cdots, n ;\left|\mathscr{E}_{i}\right|=p_{i}, i=n+1, \cdots, 2 n \\
\xi_{i} & =1, i=1, \cdots, n ; \xi_{i}=0, i=n+1, \cdots, 2 n .
\end{aligned}
$$

Substituting these in the above formula we get:

$$
\begin{aligned}
\phi_{G}(2)= & \left(p_{1}^{2\left(r_{1}-1\right)}-p_{1}^{r_{1}-1}\right)\left(p_{2}^{2\left(r_{2}-1\right)}-p_{2}^{r_{2}-1}\right) \cdots\left(p_{n}^{2\left(r_{n}-1\right)}-p_{n}^{r_{n}-1}\right) \\
& \times\left(p_{1}^{2}-1\right)\left(p_{2}^{2}-1\right) \cdots\left(p_{n}^{2}-1\right) \\
= & p_{1}^{r_{1}-1} p_{2}^{r_{2}-1} \cdots p_{n}^{r_{n}-1}\left(p_{1}^{2}-1\right)\left(p_{2}^{2}-1\right) \cdots\left(p_{n}^{2}-1\right) \\
& \times\left(p_{1}^{r_{1}-1}-1\right)\left(p_{2}^{r_{2}-1}-1\right) \cdots\left(p_{n}^{r_{n}-1}-1\right) .
\end{aligned}
$$

Since $r_{i} \geqq 2$, this shows that $\phi_{G}(2)>0$ and so $G$ can be generated by two elements.

3. Minimal-not-Sylow tower groups. In this section we will 
study groups $G$ with the property that every proper subgroup of $G$ is a Sylow tower group (STG) but $G$ is not. We call such groups minimal-not-STG. Minimal-not-nilpotent groups are known to be solvable (Schmidt) and their structure is completely known. If $G$ is a minimal-not-nilpotent group, then $|G|=p^{a} q^{b}, p \neq q ; P \triangleleft G$ where $P$ is a Sylow $p$-subgroup of $G$; $S_{q}$-subgroups of $G$ are cyclic. A group $G$ is called $p$-nilpotent if it has a normal $p$-complement. Itô showed that if $G$ is a minimal-not-p-nilpotent group, then every proper subgroup of $G$ is actually nilpotent and so $G$ has the above structure.

THeORem 3.1. Let $<$ be a total ordering on the set of all primes. Suppose that every proper subgroup of $G$ is a STG of type $<$ but $G$ is not.

Then: (1) $G$ is solvable.

(2) Indeed, $G$ is a minimal-not-nilpotent group.

(This theorem has already been proved by John S. Rose in "Finite Groups with Prescribed Sylow Groups": London Math, Soc., 3) 16 (1966), 588.)

Proof. Let $|G|=\prod_{i=1}^{n} p_{i}^{n_{i}}$ where $p_{1}<p_{2}<\cdots<p_{n} ; n_{i} \geqq 1$. Since every proper subgroup of $G$ is a STG of this type so every proper subgroup of $G$ is $p_{n}$-nilpotent. If $G$ is also $p_{n}$-nilpotent, then there is a $K \triangleleft G$ with $p_{n} \nmid|K|$ and $|G / K|=p_{n}^{*}$. Since $K<G$, so $K$ is a STG of type < and, therefore, so is $G$. Hence $G$ is not $p_{n}$-nilpotent, i.e., $G$ is a minimal-not- $p_{n}$-nilpotent group and, therefore, by Itô's theorem it is a minimal-not-nilpotent group.

We consider now a more general situation. Suppose every proper subgroup of $G$ is a STG of some type (not necessarily all of the same type), but $G$ is not. Then what can we say about $G$ ? It turns out that in this case $G$ even does not have to be solvable. For example, the alternating group $A_{5}$ has this property.

In the proof of Theorem 2.2 we obtained some properties of critical groups. Our main goal in proving that theorem was to show that critical groups are generated by two elements. One can actually determine all such groups from the information obtained about them in the proof of Theorem 2.2 and some more work. This was done by Thompson and more recently by Hawkes, and the result is that if $G$ is a solvable critical group, then $G$ is one of the following groups:

(1) $|G|=p^{a} q^{b}, G$ has a unique minimal normal subgroup $F(G)$ and $G / F(G)$ is a minimal-not-nilpotent group.

(2) $G=U_{1} \times U_{2} \times \cdots \times U_{n}$, where $U_{i}=\left\langle x_{i}\right\rangle A_{i+1}, i=1,2, \cdots, n$ with $A_{i} \cdot \triangleleft G,\left|A_{i}\right|=p_{i}^{r_{i}-1}$ and $\left|x_{i}\right|=p_{i}$ and $|G|=\prod_{i=1}^{n} p_{i}^{r_{i}}$.

Theorem 3.2. Let $G$ be a minimal-not-STG. If $G^{*}=G / \phi(G)$, 
then $G^{*}$ is one of the following groups:

(a) groups (1) or (2) above.

(b) $\operatorname{PSL}(2, p), p>3, p^{2}-1 \not \equiv 0$ (5) and $p^{2}-1 \not \equiv 0$ (16).

(c) $\operatorname{PSL}\left(2,2^{q}\right), q$ a prime.

(d) $\operatorname{PSL}\left(2,3^{q}\right), q$ odd prime.

(e) $S z(q), q=2^{p}, p$ odd prime.

Proof. Case 1. $G$ is solvable.

In this case $G^{*}$ is solvable and since $\phi\left(G^{*}\right)=1$ so every proper homomorphic image of $G^{*}$ is a STG. So $G^{*}$ is a solvable critical group and, therefore, is one of the groups (1) or (2).

Case 2. $G$ is not solvable.

In this case $G^{*}$ must be simple and since all proper subgroups of $G^{*}$ are solvable, so $G^{*}$ is a minimal simple group and is, therefore, one of the following groups:

(i) $\operatorname{PSL}(2, p), p>3, p^{2}-1 \not \equiv 0(5)$.

(ii) $\operatorname{PSL}\left(2,2^{q}\right), q$ any prime.

(iii) $\operatorname{PSL}\left(2,3^{q}\right), q$ odd prime.

(iv) $\operatorname{PSL}(3,3)$.

(v) $S z(q), q=2^{p}, p$ odd prime.

However, not all of these are minimal-not-STG's, e.g., PSL(2, 7) has a subgroup isomorphic to the symmetric group $\Sigma_{4}$ on four letters and so it has a proper subgroup which has no Sylow tower. In order to determine which of these groups are actually minimal-not-STG's we need the following theorem of Dickson (cf. [7], p. 213):

THEOREM (Dickson). The group PSL(2, $\left.p^{f}\right)$ has only the following subgroups:

(a) elementary Abelian.

(b) cyclic groups of order $z$, where $z \mid\left(\left(p^{f} \pm 1\right) / k\right), k=\left(p^{f}-1,2\right)$.

(c) dihedral groups of order $2 z, z$ as above.

(d) $A_{4}$ for $p>2$ or $p=2$ and even $f$.

(e) semi-direct product of an elementary Abelian p-group of order $p^{m}$ by a cyclic group of order $t$, where $t \mid p^{m}-1$.

(f) $\Sigma_{4}$ if and only if $p^{2 f}-1 \equiv 0(16)$.

(g) $A_{5}$ if and only if $p=5$ or $p^{2 f}-1 \equiv 0(5)$.

(h) groups $P S L\left(2, p^{m}\right)$ for $m \mid f$ and $P G L\left(2, p^{m}\right)$ for $2 m \mid f$.

Among these only (f), (g), and (h) do not have Sylow towers. So we check the list (i)-(iii) of minimal simple groups for these conditions. None of them can have subgroups of type (g) and (h) as they are minimal simple. So we only have to check the condition (f). We get: PSL $(2, p), p>3, p^{2}-1 \not \equiv 0(5)$ is a minimal-not-STG if and only 
if $p^{2}-1 \not \equiv 0(16)$.

$\operatorname{PSL}\left(2,2^{q}\right), q$ any prime $i s$ a minimal-not-STG, since $2^{q}-1 \not \equiv 0(16)$.

Let $G=\operatorname{PSL}\left(2,3^{q}\right), q$ odd prime. Then

$$
\begin{aligned}
p^{2 f}-1=3^{2 q}-1=9^{q}-1 & =(9-1)\left(9^{q-1}+9^{q-2}+\cdots+9+1\right) \\
& =8\left(9^{q-1}+9^{q-2}+\cdots+9+1\right) .
\end{aligned}
$$

The number of terms in the second factor on the right-hand side is $q$ and, therefore, is odd. Since each summand in that factor is also odd, we get that $p^{2 f}-1 \not \equiv(16)$ and so PSL $\left(2,3^{q}\right)$ is a minimal-not-STG.

Claim: $\operatorname{PSL}(3,3)$ has a subgroup isomorphic to $\Sigma_{4}$.

Since the only third root of 1 in $G F(3)$ is 1 , so

$$
\operatorname{PSL}(3,3) \approx \mathrm{SL}(3,3) \text {. }
$$

Let $V$ be a 3 -dimensional vector space over $K=\mathrm{GF}(3)$ with basis $\left\{\alpha_{1}, \alpha_{2}, \alpha_{3}\right\}$. We set:

$$
H=\{I, \operatorname{diag}(2,2,1), \operatorname{diag}(2,1,2), \operatorname{diag}(1,2,2)\} .
$$

Clearly, $H$ is an Abelian subgroup of order 4 of $\mathrm{SL}(3,3)$ and is a four group. Define $N$ by:

$$
N=\left\{g \in \mathrm{GL}(3,3) \mid \alpha_{i} g=\varepsilon \alpha_{\sigma(i)} \forall \sigma \in \Sigma_{3}, \varepsilon\right\}=\left\{\begin{array}{lll}
1, & \text { if } & \sigma \in A_{3} \\
2, & \text { if } & \sigma \in \Sigma_{3}-A_{3}
\end{array}\right\} .
$$

It can easily be seen that $N$ is actually a subgroup of $\mathrm{SL}(3,3)$, isomorphic to $\Sigma_{3}$. It consists of the following matrices:

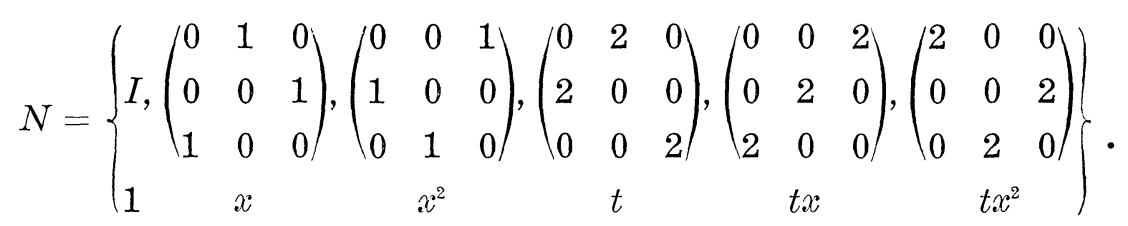

So we have $H, N \leqq \operatorname{PSL}(3,3)=G$. If $g \in N$, then $\alpha_{i} g=\varepsilon \alpha_{\sigma(i)}$. Let $h \in H$, so $h=\operatorname{diag}\left(b_{1}, b_{2}, b_{3}\right), b_{i} \neq 0$. We have

$$
\alpha_{\sigma(i)} h^{g}=\alpha_{\sigma(i)}\left(g^{-1} h g\right)=\varepsilon^{-1} \alpha_{i}(h g)=\varepsilon^{-1} b_{i}\left(\alpha_{i} g\right)=b_{i} \alpha_{\sigma(i)}, i=1,2,3 .
$$

So $h^{g}$ is diagonal with determinant 1 (= the determinant of $h$ ), i.e., $h^{g} \in H$. So $N \leqq N_{G}(H)$. Hence, $L=N H$ is a subgroup of $G$ isomorphic to $\Sigma_{4}$ and the claim is established. This shows that PSL $(3,3)$ is not a minimal-not-STG.

Finally, we consider the Suzuki groups $S z(q), q=2^{p}$, $p$ odd prime. These are simple groups of order $q^{2}(q-1)\left(q^{2}+1\right)$ and Suzuki has shown that they can only have the following subgroups [8]: 
(a) Frobenius groups $H$ of order $q^{2}(q-1), H=Q K, Q \triangleleft H, Q \epsilon$ $\operatorname{Syl}_{q}(H), K$ cylic of order $q-1$.

(b) dihedral groups of order $q(q-1)$.

(c) cyclic groups $A_{i}, i=1,2$ of orders $q \pm r+1$, where $r^{2}=2 q$.

(d) $B_{i}=N\left(A_{i}\right), i=1,2$ of orders $4(q \pm r+1)$.

(e) $S z(s)$, where $q$ is a power of $s$.

But (e) cannot happen since we are considering minimal simple groups. The groups of type (a), (b), and (c) are clearly STG's. Since

$$
\begin{aligned}
|G|=q^{2}(q-1)\left(q^{2}+1\right) & =q^{2}(q-1)\left(q^{2}+1+2 q-2 q\right) \\
& =q^{2}(q-1)\left[(q+1)^{2}-2 q\right] \\
& =q^{2}(q-1)(q+1+r)(q+1-r),
\end{aligned}
$$

and since $q$ and $r$ are powers of 2 , so $A_{i}$ are Hall subgroups of $G$. So they are Hall subgroups of $B_{i}$. This means each $B_{i}$ is a STG being the extension of a cyclic group by an Abelian group. So all subgroups of $S z(q)$ are STG's and we get that $S z(q), q=2^{p}, p$ odd prime is a minimal-not-STG. This completes the proof of Theorem 3.2.

\section{CHAPTER II}

\section{On Generalized Sylow Tower Groups of Derr}

1. Saturation of the formation of all generalized Sylow tower groups of a given complexion. Generalized Sylow Tower Groups (GSTG) were introduced by J. Derr in [4]. If $G$ is a Sylow tower Group (STG), say of type $p_{1}<p_{2}<\cdots<p_{n}$, then there is a set $\left\{G_{p_{1}}, G_{p_{2}}, \cdots, G_{p_{n}}\right\}$ of Sylow subgroups of $G$ such that if $i>j$, then $G_{p_{i}} \leqq N\left(G_{p_{j}}\right)$. Derr generalizes this property of STG's to get:

Definition. A group $G$ is a GSTG if there is a complete set $\left\{G_{p}|p||G|\right\}$ of Sylow subgroups of $G$ satisfying:

$(\mathrm{N})$ : for $p \neq q$, either $G_{p} \leqq N\left(G_{q}\right)$ or $G_{q} \leqq N\left(G_{p}\right)$.

Now, if $G$ is a GSTG and $p$ and $q$ are two distinct primes such that, say, $G_{p} \leqq N\left(G_{q}\right)$, we write $p R q$. This defines a relation $R$ on the set of all primes and we say that $G$ is a GSTG of complexion $R$. It can be seen that subgroups and homomorphic images of GSTG's are also GSTG's and, in fact, that the class of all GSTG's of a given complexion $R$ is a formation [4]. We will prove, in this section, that this formation is actually saturated (Theorem 1.2).

Theorem 1.1. If $G$ is a GSTG, then $l_{p}(G) \leqq 1$, for all primes $p$. 
Proof. Let $G$ be a minimal counter-example. So $G$ is a GSTG and there is a prime $q$ such that $l_{q}(G)>1$. Furthermore, if $H$ is a GSTG and $|H|<|G|$, then $l_{p}(H) \leqq 1$, for all $p$. $G$ has the following properties:

(1) $G$ has a unique minimal normal subgroup $N$. If $N_{1}$ and $N_{2}$ are two distinct minimal normal subgroups of $G$, then $N_{1} \cap N_{2}=1$. Since $G / N_{i}, i=1,2$ are GSTG's of smaller orders than $|G|$, so $l_{q}\left(G / N_{i}\right) \leqq 1, i=1,2$. Hence,

$$
l_{q}(G)=l_{q}\left(G / N_{1} \cap N_{2}\right)=\operatorname{Max}\left\{l_{q}\left(G / N_{1}\right), l_{q}\left(G / N_{2}\right)\right\} \leqq 1,
$$

a contradiction. This proves (1).

(2) $\phi(G)=1$.

This follows from the minimality of $G$ and the fact that $l_{q}(G)=$ $l_{q}(G / \phi(G))$ (cf. [7], p. 689).

(3) $O_{q^{\prime}}(G)=1, N$ is a $q$-group and $N=C(N)$.

If $O_{q^{\prime}}(G)>1$, then $1<l_{q}(G)=l_{q}\left(G / O_{q^{\prime}}(G)\right) \leqq 1$, a contradiction. So $O_{q^{\prime}}(G)=1$. Since $G$ is solvable, $|N|$ is power of some prime, so $N$ must be a $q$-group. It follows from (2) that there is a maximal subgroup $M$ of $G$ such that $N \geqq M$, and so $M$ is a complement for $N$ in $G$. So

$$
G=M N, \quad M \cap N=1 .
$$

Since $N$ is Abelian, $N \leqq C(N)$. So $C(N)=(C(N) \cap M) N$, by Dedekind identity. Since $C(N) \cap M$ is normal in $M$ and is centralized by $N, C(N) \cap M \triangleleft G$. Thus, if $C(N) \cap M>1$, then by (1), $N \leqq$ $C(N) \cap M$. So $N \leqq M$, a contradiction. Hence, $C(N) \cap M=1$ and, therefore, $C(N)=N$.

(4) Contradiction.

Let $\left\{G_{p}|p \| G|\right\}$ be a complete set of Sylow subgroups of $G$ satisfying the property $(N)$. If for all primes $p, p \neq q$ we have $G_{p} \leqq N\left(G_{q}\right)$, then $G_{q} \triangleleft G$ and so $l_{q}(G)=1$, a contradiction. So there is a prime $p, p \neq$ $q$ such that $G_{p} \geqq N\left(G_{q}\right)$. But then by $(N)$ we have $G_{q} \leqq N\left(G_{p}\right)$. Now, $N \leqq G_{q} \leqq N\left(G_{p}\right)$. So $N$ and $G_{p}$ are two normal subgroups of $N\left(G_{p}\right)$ of coprime orders. So $G_{p} \leqq C(N)=N$. But this cannot happen as $N$ is a $q$-group and $p \neq q$.

THEOREM 1.2. If $G / \phi(G)$ is a GSTG of a given complexion $R$, then so is $G$.

Proof. Let $G$ be a minimal counter example. We get:

(1) $G$ has a unique minimal normal subgroup $N,|N|=r^{*}, r$ a prime.

If $N_{i}, i=1,2$ are two distinct minimal normal subgroups of $G$, then 
$N_{1} \cap N_{2}=1$. Since $\phi(G) N_{i} / N_{i} \leqq \phi\left(G / N_{i}\right)$, we get:

$G / N_{i} / \phi\left(G / N_{i}\right)$ is a homomorphic image of $G / N_{i} / \phi(G) N_{i} / N_{i} \approx G / \phi(G) N_{i}$, and this in turn is a homomorphic image of $G / \phi(G)$ which is a GSTG of complexion $R$. So $G / N_{i} / \dot{\phi}\left(G / N_{i}\right), i=1,2$, are GSTG's of complexion $R$. So by minimality of $G$ we get that $G / N_{i}, i=1,2$ are GSTG's of complexion $R$. But then so is $G \approx G / N_{1} \cap N_{2}$, a contradition. This proves (1). Let $|N|=r^{*}$ for some prime $r$. We have $N \leqq \phi(G)$, since $\phi(G)>1$ and $N$ is the unique minimal normal subgroup of $G$.

(2) $G$ has a normal Sylow $r$-subgroup.

It follows from (1) that $O_{r^{\prime}}(G)=1$, so $O_{r^{\prime} r}(G)=O_{r}(G)$. On the other hand, since $G / \phi(G)$ is a GSTG, Theorem 1.1 gives $l_{r}(G / \phi(G))=1$. So $l_{r}(G)=1$. This shows that $O_{r}(G)$ is the normal $S_{r}$-subgroup of $G$.

(3) Contradiction.

By Schur-Zassenhaus, $G=R K, R \cap K=1$, where $R$ is the $S_{r}$-subgroup of $G$. Since $K \approx G / R$, by minimality of $G, K$ is a GSTG of complexion $R$. Let $\left\{K_{p}|p||G|\right\}$ be a complete set of Sylow subgroups of satisfying $(N)$. Then

$$
\left\{R, K_{p}|p||K|\right\}
$$

is a complete set of Sylow subgroups of $G$ and clearly $G$ is a GSTG. To prove it has complexion $R$, we only need to show that if in $G / \phi(G)$, in addition to $p R r$ (which must hold), it is also true that $r R p$ for some $p \neq r$ then a similar relation holds in $G$. The assumption on $G / \phi(G)$ implies that in addition to

$$
K_{p} \dot{\phi}(G) / \dot{\phi}(G) \leqq N_{G \mid \phi(G)}(R / \dot{\phi}(G)),
$$

we also have

$$
R / \dot{\phi}(G) \leqq N_{G \mid \phi(G)}\left(K_{p} \phi(G) / \dot{\phi}(G)\right) .
$$

But then $R / \phi(G)$ and $K_{p} \phi(G) / \phi(G)$ centralize each other, i.e., [R, $\left.K_{p} \phi(G)\right] \leqq \phi(G)$, and so $\left[R, K_{p}\right] \leqq \phi(G)$. We show $\phi(G)=\phi(R)$ :

Note that $\phi(R)=\phi(F(G)) \leqq \phi(G)$. Since $K$ acts on the elementary abelian $r$-group $R / \phi(R)$ and $(|K|, r)=1$, by Maschke's theorem $R / \phi(R)$ is a completely reducible $K$-module; and since $\phi(G) / \phi(R)$ is a proper $K$-submodule of $R / \phi(R)$ we get that $\dot{\phi}(G)=\dot{\phi}(R)$. This yields $\left[R, K_{p}\right] \leqq \phi(R)$. Since $K_{p}$ acts on $R$ and $\left(\left|K_{p}\right|,|R|\right)=1$, we get $R=$ $\left[R, K_{p}\right] C_{R}\left(K_{p}\right)=\phi(R) C_{R}\left(K_{p}\right)$.

So $R=C_{R}\left(K_{p}\right)$ and, therefore, $\left[R, K_{p}\right]=1$. From this it is obvious that $R \leqq N_{G}\left(K_{p}\right)$, i.e., $r R p$ holds in $G$.

We have shown that $G$ is GSTG of complexion $R$, contradicting our assumption.

2. Structure of GSTG's. In this section we find a way of con- 
structing GSTG's of a given complexion and study the groups which are GSTG's but not STG's. In particular, we find all such groups whose orders are divisible by precisely three distinct primes.

Let $R$ be a fixed relation on the set of all primes. We denote by $\mathscr{S}^{*}$ the class of all GSTG's of complexion $R$. Let $\mathscr{S}$ be the smallest class of groups containing all prime-power groups such that:

(1) $\mathscr{S}$ is closed under subdirect products (i.e., subgroups of direct products).

(2) If $H \in \mathscr{S}$ is a $p$-group and $K \in \mathscr{S}$ is a $p^{\prime}$-group and $q R p$ for all $q \in \pi(K)$, then the semi-direct product group $[H] K \in \mathscr{S}$.

\section{THEOREM 2.1. $\mathscr{S}=\mathscr{S}^{*}$.}

Proof. $\mathscr{S}^{*}$ contains all prime-power groups and satisfies the conditions (1) and (2); and since $\mathscr{S}$ is the smallest class with these properties, we get that $\mathscr{S} \cong \mathscr{S}^{*}$.

To prove the converse, suppose by way of contradiction that $\mathscr{S}^{*} \nsubseteq \mathscr{S}_{\text {. }}$ Choose $G$ minimal with the property that $G \in \mathscr{S}^{*}$, but $G \notin \mathscr{S}$.

If $N_{1}$ and $N_{2}$ are two distinct minimal normal subgroups of $G$, then $N_{1} \cap N_{2}=1$. $G / N_{i}, i=1,2$ are GSTG's and so by minimality of $G$, $G / N_{i} \in \mathscr{S}, i=1,2$. So $G \in \mathscr{S}$, by (1). Hence, $G$ has a unique minimal normal subgroup $N$ with $|N|=p^{*}$ and so $O_{p^{\prime}}(G)=1$. Since $l_{p}(G) \leqq$ 1 by Theorem 1.1, so $G$ has a normal $S_{p}$-subgroup $P$. But then by Schur-Zassenhaus,

$$
G=P K, \quad P \cap K=1, \quad P \triangleleft G .
$$

Now $K \in \mathscr{S}^{*}$, so by minimality of $G, K \in \mathscr{S}$ and since $P \in \mathscr{S}$ and $q R p$ for all $q \in \pi(K)$, so by (2), $G=[P] K \in \mathscr{S}$, a contradiction. So $\mathscr{S}^{*} \subseteq \mathscr{S}$, and the proof of Theorem 2.1 is complete.

One may ask if there are GSTG's which are not STG's; and if so, is it possible to obtain all such GSTG's from STG's with certain operations. Clearly, if the order of a GSTG is divisible by only two distinct primes, then it is also a STG. In Theorem 2.3 we will find all GSTG's whose orders are divisible by precisely 3 distinct primes and are not STG's.

Definition. A group $X$ is called a G-group if it is a GSTG but not a STG. We say $X$ is a $G_{n}$-group if $X$ is a $G$-group and its order is divisible by exactly $n$ distinct primes.

Lemma 2.2. Suppose the group $G$ is of the form $[[R] Q] P$, where $P, Q$, and $R$ are $S_{p}, S_{q}$, and $S_{r}$-subgroups of $G$ respectively. Suppose, 
in addition, that $R \leqq N(P)$. Then $G$ is contained in the direct product of groups of the form $\left[Q_{1}\right] P_{1}$ and $\left[R_{1}\right] Q_{2}$ where $P_{i}, Q_{i}$, and $R_{i}$ are $p$, $q$, and r-groups respectively.

Proof. Let $G$ be a minimal counter-example. If $M, N \cdot \triangleleft G$ and $M \neq N$, then $M \cap N=1$. Since $G / M$ and $G / N$ satisfy the hypothesis, we get

$$
G / M \leqq H_{1} \times K_{1} \text { and } G / N \leqq H_{2} \times K_{2}
$$

where $H_{i}$ are of the form $\left[Q_{j}\right] P_{j}$ and $K_{i}$ are of the form $\left[R_{k}\right] Q_{k}$. But then since $G \leqq G / M \times G / N$, we get that $G$ also has the same form, a contradiction. So $G$ has a unique minimal normal subgroup $N$. $N$ must be an $r$-group as $G$ has a normal $S_{r}$-subgroup. Since $R$ and $P$ normalize each other, they must centralize each other and so we have $[R, P]=1$. Also, since $[R, Q, P] \leqq[R, P]=1$ and $[P, R, Q]=1$, by 3-subgroup lemma, we get that $[Q, P, R]=1$, i.e., $[Q, P]$ centralizes R. Hence, $[Q, P]$ is normalized by $P, Q$, and $R$ and so $[Q, P] \triangleleft G$. But $[Q, P]$ is a $q$-group and $O_{q}(G)=1$. So $[Q, P]=1$. Thus, $P$ is centralized by $R$ and $Q$ and we have

$$
G=P \times R Q=P \times[R] Q .
$$

This contradicts the assumption that $G$ was a counter-example.

THEOREM 2.3. If $G$ is a $G_{3}$-group, then $G$ is isomorphic to a subgroup of $H=\left[Q_{1}\right] P_{2} \times\left[R_{1}\right] Q_{2} \times\left[P_{1}\right] R_{2}$, where $P_{i}, Q_{i}$, and $R_{i}$ are $p$, $q$, and r-groups respectively.

Proof. $G$ cannot have a normal Sylow subgroup. Because if it did, the quotient being a GSTG with order divisible by exactly two distinct primes would have to be a STG and this forces $G$ to be a STG. So if $p, q$, and $r$ are the three distinct prime divisors of $G$, then $G$ must have complexion $R$, where

$$
p R q, q R r, \text { and } r R p \text {. }
$$

By Theorem 2.1 $G$ is obtained from prime-power groups by applying the following operations:

(1) taking subgroups of direct products;

(2) extension of a $p$-group by a GSTG $H$ of complexion $R$, where $p \notin \pi(H)$.

So in order to construct our group $G$ we start with prime-power groups $P_{i}, Q_{i}$, and $R_{i}$ and apply (1) first. We get a nilpotent group $H$. If $|H|$ is divisible by $p, q$, and $r$, then we cannot apply (2) to it any more and we must get a nilpotent group which is not the case. 
So we may assume that $|H|$ is divisible by 2 primes, i.e., $H$ is one of the following groups
(a) $P_{1} \times Q_{2}$
(b) $Q_{1} \times R_{2}$
(c) $R_{1} \times P_{2}$.

Applying (2) to these, we get:
(a) $\left[R_{3}\right]\left(P_{1} \times Q_{2}\right)$
(b) $\left[P_{3}\right]\left(Q_{1} \times R_{2}\right)$
(c) $\left[Q_{3}\right]\left(R_{1} \times P_{2}\right)$.

None of these could be our group $G$, as they each have a normal Sylow subgroup. If these were to be constituents of $G$, they must be GSTG's of complexion $R$ and so we have additional properties:

$$
R_{3} \leqq N\left(P_{1}\right), \quad P_{3} \leqq N\left(Q_{1}\right), \quad Q_{3} \leqq N\left(R_{1}\right) .
$$

So the group in (a) satisfies the hypothesis of Lemma 2.2. The groups in (b) and (c) satisfy similar hypothesis; and since we can now only apply (1) to (a), (b), and (c), Lemma 2.2 shows that $G$ must be of the required form.

We could start with the prime-power group $P_{i}, Q_{i}$, and $R_{i}$ and apply (2) first. We get the following groups:
$(*)$
$\left[Q_{1}\right] P_{2}, \quad\left[R_{1}\right] Q_{2}, \quad\left[P_{1}\right] R_{2}$.

Applying (1) to these yields a group of the required form. If we apply (2) once more to $\left(^{*}\right)$, we get:

$$
\left[R_{3}\right]\left(\left[Q_{1}\right] P_{2}\right), \quad\left[P_{3}\right]\left(\left[R_{1}\right] Q_{2}\right), \quad\left[Q_{3}\right]\left(\left[P_{1}\right] R_{2}\right) .
$$

As before, each of these groups has a normal Sylow subgroup and, hence, could not be our group $G$. If they were constituents of $G$, we must have

$$
R_{3} \leqq N\left(P_{2}\right), \quad P_{3} \leqq N\left(Q_{2}\right), \quad Q_{3} \leqq N\left(R_{2}\right) .
$$

Therefore, these groups satisfy Lemma 2.2, and so $G$ must have the required form.

We have considered all the possibilities and since $|G|$ is divisible by only three distinct primes, this process terminates here and the theorem is proved.

3. Minimal-not-GSTG's and critical groups. In section three of Chapter I we saw that if every proper subgroup of a group $G$ is a STG of the same type, but $G$ is not, then $G$ must be solvable and, indeed, it has to be a minimal-not-nilpotent group. The similar statement is not true for GSTG's, i.e., if every proper subgroup of a group $G$ is a GSTG of the same complexion, but $G$ is not, then $G$ does not have to be solvable (Proposition 3.1). 
DEFINITION. $G$ is a minimal-not-GSTG if every proper subgroup of $G$ is a GSTG but $G$ is not. If every proper subgroup of $G$ is a GSTG of the same complexion $R$ but $G$ is not, we say that $G$ is a minimal-not-R-GSTG. Here $R$ is a fixed relation on the set of primes.

Proposition 3.1. $G=P S L(2,27)$ is a non-solvable minimal-not$R$-GSTG, where $R$ is the following relation:

$2 R 7,2 R 13,3 R 2,13 R 3$, and $p R q$

for $p, q$ primes other than 2, 3, 7, and 13.

Proof. $G$ is a minimal simple group of order

$$
\frac{27 \times 26 \times 28}{2}=2^{2} \times 3^{3} \times 7 \times 13,
$$

and as we saw in section three of Chapter I, it can only have the following subgroups:

1. elementary Abelian.

2. cyclic groups of order $z$, where $z \mid(27 \pm 1) / 2=13$ or 14 .

3. dihedral groups of order $2 z, z$ as above.

4. $A_{4}$.

5. semi-direct product of an elementary Abelian 3-group of order $3^{m}$ by a cyclic group of order $t$, where $t \mid(27-1)$.

The proper subgroups of types $1,2,3$, and 4 are clearly GSTG's of complexion $R$.

Claim. $G$ does not have a subgroup which is the semi-direct product of an elementary Abelian 3-group by an element of order 2 .

If it did, it would have a subgroup isomorphic to $\Sigma_{3}$, i.e., $G$ would have a subgroup $H=\left\langle x, y \mid x^{2}=y^{3}=1, y^{x}=y^{-1}\right\rangle . \quad y$ is a 3-element so $y \in P$, some Sylow 3-subgroup of $G$. But then $1 \neq y^{x}=$ $y^{-1} \in P$. So $1 \neq y^{x} \in P \cap P^{x}$. But distinct $S_{p}$-subgroups of $\operatorname{PSL}\left(2, p^{f}\right)$ have trivial intersections [7, p. 191]. So $P=P^{x}$, i.e., $x \in N(P)$. But $|N(P)|=13 \times 27$, an odd number. This establishes the claim.

The only other possibility for a proper subgroup of $G$ is the semidirect product of an elementary Abelian 3-subgroup by an element of order 13, which is a GSTG of complexion $R$, as $13 R 3$.

Proposition 3.2. The groups PSL $\left(2,3^{q}\right)$, $q$ odd prime, are minimal-not-R-GSTG's, where the relation $R$ is defined as follows:

$2 R p$, all primes $p$ with $p \neq 3$

$3 R 2$

$r R 3$, all primes $r$ with $r \mid\left(3^{q}-1\right)$ and $r \neq 2$ 


\section{sRt, all primes $s$ and $t$ other than 2 and 3 .}

Proof. Again, looking at the list of subgroups of $\operatorname{PSL}\left(2,3^{q}\right)$, the dihedral, cyclic, and elementary Abelian subgroups are GSTG's of complexion $R$, and so is $A_{4}$. For the same reason as before, $\operatorname{PSL}\left(2,3^{q}\right)$ has no subgroups isomorphic to $\Sigma_{3}$ and, therefore, the only other possibility for a subgroup of $\operatorname{PSL}\left(2,3^{q}\right)$ is the semi-direct product of an elementary Abelian 3-group by a cyclic group of order $t$, where $t \mid 3^{q}-1$ and $t$ is odd. These type of subgroups are clearly GSTG's of complexion $R$. This completes the proof of the proposition.

Definition. $G$ is called $R$-critical, $R$ a fixed relation on the set of all primes, if every proper subgroup and every proper homomorphic image of $G$ is a GSTG of complexion $R$ but $G$ is not.

THeOREM 3.3. If $G$ is a non-solvable $R$-critical group, then $G$ is isomorphic to $P S L\left(2,3^{q}\right), q$ odd prime and $R$ is the relation defined in Proposition 3.2.

Proof. Since every proper subgroup and homomorphic image of $G$ is solvable and $G$ is not, so $G$ must be simple. In fact, $G$ is a minimal simple group and so is one of the following groups:

1. $\operatorname{PSL}(2, p), p>3, p^{2}-1 \neq 0(\bmod 5)$

2. $\operatorname{PSL}\left(2,2^{q}\right), q$ any prime

3. $\operatorname{PSL}\left(2,3^{q}\right), q$ odd prime

4. $\operatorname{PSL}(3,3)$

5. $\mathrm{Sz}\left(2^{p}\right), p$ odd prime.

We claim:

(a) $G$ cannot be $\operatorname{PSL}(2, p), p>3, p^{2}-1 \not \equiv 0(\bmod 5)$. Because this group has a subgroup isomorphic to $A_{4}$. It also has dihedral subgroups of order $p-1$ and $p+1$. Since $p>3$, one of these numbers is divisible by 3 and thus the corresponding dihedral group has a subgroup isomorphic to $\Sigma_{3}$. This means PSL(2, $\left.p\right), p>3$ has subgroups isomorphic to $A_{4}$ and $\Sigma_{3}$ which are GSTG's of opposite complexion.

(b) $G$ cannot be $\operatorname{PSL}\left(2,2^{q}\right), q$ a prime.

The normalizer of a $S_{2}$-subgroup $P$ of $\operatorname{PSL}\left(2,2^{q}\right)$ is the semi-direct product of $P$ by a cyclic group of order $2^{q}-1$. Let $r$ be a prime divisor of $2^{q}-1$. Then $N(P)$ has a subgroup which is the semidirect product of $P$ by $\langle r\rangle$. On the other hand, PSL $\left(2,2^{q}\right)$ has a dihedral subgroup of order $2\left(2^{q}-1\right)$. So it has a dihedral subgroup of order $2 r$. This shows that PSL $\left(2,2^{q}\right)$ has two subgroups which are GSTG's of opposite complexion.

(c) $G$ cannot be $\operatorname{PSL}(3,3)$. 
As we saw before, $\operatorname{PSL}(3,3)$ has a subgroup isomorphic to $\Sigma_{4}$ which is not a GSTG.

(d) $G$ cannot be $S z(q), q=2^{q}, p$ odd prime.

$S z(q)$ has a subgroup $H$ of order $q^{2}(q-1)$ which is a Frobenius group with normal $S_{2}$-subgroup of order $q^{2}$. Let $r$ be a prime divisor of $q-1$. Then $H$ has a subgroup which is the semi-direct product of $S_{2}$-subgroup of $H$ with an element of order $r$. On the other hand, $S z(q)$ has a dihedral subgroup of order $2(q-1)$ and thus one of order $2 r$. So $S z(q)$ has two subgroups which are GSTG's of opposite complexion.

So the only possibility for $G$ is $\operatorname{PSL}\left(2,3^{q}\right), q$ odd prime, which by Proposition 3.2 is a minimal-not- $R$-GSTG and since it is simple, also an $R$-critical group.

Theorem 3.4. If $G$ is an R-critical group, then it must be one of the following groups:

(1) $\operatorname{PSL}\left(2,3^{q}\right), q$ odd prime.

(2) $|G|=p^{a} q^{b}$, indeed, $G$ is a minimal-not-nilpotent group.

Proof. If $G$ is nonsolvable, then by Theorem 3.3, $G$ is isomorphic to $\operatorname{PSL}\left(2,3^{q}\right), q$ odd prime. So we may assume that $G$ is solvable. Let $\left\{G_{p_{i}} \mid i=1,2, \cdots, r\right\}$ be a Sylow basis for $G$. If $r>2$, then $G_{p_{i}} G_{p_{j}}$, $i \neq j$ is a proper subgroup of $G$ and so is a GSTG of complexion $R$. Since this is true for all $i$ and $j$, it follows that $G$ is a GSTG of complexion $R$. So $r=2$ and $|G|=p^{a} q^{b}$.

Say the relation $R$ is so that $p R q$. Since all proper subgroups of $G$ are GSTG's of complexion $R$, so they are STG's whose orders are divisible by $p$ and $q$ only and they all have a normal $p$-complement. So by Itô's theorem either $G$ is $p$-nilpotent or it is a minimalnot-nilpotent group. But $G$ cannot be $p$-nilpotent, otherwise it would be a GSTG of complexion $R$. This completes the proof of this theorem.

THEOREM 3.5. If $G$ is a minimal-not-R-GSTG, then $G^{*}=G / \dot{\phi}(G)$ is one of the groups (1) or (2) in Theorem 3.4.

Proof. Every proper subgroup of $G^{*}$ being a homomorphic image of a proper subgroup of $G$ is a GSTG of complexion $R$. If $1 \neq N^{*} \triangleleft$ $G^{*}$, then since $N^{*} \notin \dot{\phi}\left(G^{*}\right)=1$, so there is $M^{*}<\cdot G^{*}$ so that $N^{*} \not$ $M^{*}$. So $G^{*}=N^{*} M^{*}$. But then

$$
G^{*} / N^{*}=M^{*} N^{*} / N^{*} \approx M^{*} / M^{*} \cap N^{*} \text {. }
$$

$M^{*}$ is a GSTG of complexion $R$ and so $G^{*} / N^{*}$ is also one such. This shows that every proper homomorphic image of $G^{*}$ is a GSTG of complexion $R$. Clearly, $G^{*}$ is not a GSTG, otherwise $G$ would be 
one also. So $G^{*}$ is an $R$-critical group and the result follows from Theorem 3.4.

DEFINITION. $G$ is a critical group (more precisely, GSTG-critical) if every proper subgroup and every proper homomorphic image of $G$ is a GSTG, but $G$ is not. Notice that here we do not require all the subgroups and homomorphic images be of the same complexion.

Theorem 3.6. Let $G$ be a critical group. Then $G$ is one of the following groups:

(a) $|G|=p^{a} q^{b}$, $G$ has a unique minimal normal subgroup $F(G)$ and $G / F(G)$ is a minimal-not-nilpotent group.

(b) $G=U_{1} \times U_{2}$, where $U_{1}=A_{1}\left\langle x_{2}\right\rangle$ and $U_{2}=A_{2}\left\langle x_{1}\right\rangle,\left|A_{1}\right|=p^{r-1},\left|A_{2}\right|=$ $q^{s-1}, o\left(x_{1}\right)=p, o\left(x_{2}\right)=q, A_{i}$ are minimal normal subgroups of $G$, and $|G|=p^{r} q^{s}$.

(c) $P S L(2, p), p>3, p^{2}-1 \not \equiv 0(5), p^{2}-1 \not \equiv 0(16)$.

(d) $\operatorname{PSL}\left(2,2^{q}\right), q$ any prime.

(e) $\operatorname{PSL}\left(2,3^{q}\right), q$ odd prime.

(f) $S z(q), q=2^{p}, p$ odd prime.

Proof. If $G$ is solvable, as in the proof of Theorem 3.4, we get that order of $G$ must be divisible by precisely two distinct primes $p$ and $q$. Since every proper subgroup of $G$ is a GSTG whose order is divisible by 2 primes so they are all STG's. Similarly, all proper homomorphic images of $G$ are STG's. This means that $G$ is a critical group with respect to STG's. So $G$ is one of groups (a) or (b), by Theorem 3.2 of Chapter I.

If $G$ is not solvable, then it must be minimal simple, and we have seen in the analysis of critical groups for STG's that $G$ must be one of the groups (c)-(f).

Finally, if $G$ is a minimal-not-GSTG, then $G^{*}=G / \phi(G)$ is a critical group and so we get the following:

Theorem 3.7. If $G$ is a minimal-not-GSTG, $G^{*}=G / \phi(G)$ is one of the groups $(a)-(f)$ in Theorem 3.6.

Theorem 3.8 gives us a final result about GSTG's.

THEOREM 3.8. If every 2-generator subgroup of $G$ is a GSTG (not necessarily all of the same complexion), then so is $G$.

Proof. Let $G$ be a minimal counter-example. If $H$ is a proper subgroup of $G$, every 2-generator subgroup of $H$ is a GSTG. So by minimality of $G, H$ is a GSTG. So $G$ is a minimal-not-GSTG. By 
Theorem 3.7, $G / \phi(G)$ is one of the groups (a)-(f) in 3.6. But all these groups can be generated by 2 elements. So $d(G / \phi(G))=2$, and thus $d(G)=2$. But every 2-generator subgroup of $G$ is a GSTG. Contradiction.

\section{REFERENCES}

1. J. L. Alperin, The construction and characterization of some classes of finite solvable groups, Arch. Math., 18 (1967), 349-354.

2. R. Baer, Sylowturmgruppen II, Math. Z., 92 (1966), 256-268.

3. R. Carter, B. Fischer, and T. O. Hawkes, Extreme classes of finite solvable groups,

J. of Algebra 9 (1968), 285-313.

4. J. Derr, Generalized Sylow tower groups, Pacific J. Math., 32 (1970), 633-642.

5. W. Gaschütz, Die Eulerche Funktion endlicher auflösbarer Gruppen, Illinois J. Math., 3 (1959), 469-476.

6. T. O. Hawkes, On the class of Sylow tower groups, Math. Z., 105 (1968), 393-398.

7. B. Huppert, Endliche Gruppen I, Springer-Verlag (1967).

8. M. Suzuki, On a class of doubly transitive groups, Ann. Math., 75 (1962), 105-145.

9. J. Thompson, Sylow series for finite groups, (unpublished).

Received October 4, 1971. I would like to express my deep appreciation and gratitude to Prof. J. L. Alperin, my thesis advisor, for his kind help and advice in the preparation of this paper which was my $\mathrm{Ph}$. D. thesis at the University of Chicago.

UNIVERSITY OF CHICAGo

AND

PAHLAVI UNIVERSITY 


\section{PACIFIC JOURNAL OF MATHEMATICS}

\section{EDITORS}

\author{
H. SAMELson \\ Stanford University \\ Stanford, California 94305 \\ C. R. HоввY \\ University of Washington \\ Seattle, Washington 98105
}

J. DugundJI

Department of Mathematics

University of Southern California

Los Angeles, California 90007

RICHARD ARENS

University of California

Los Angeles, California 90024

\section{ASSOCIATE EDITORS}
E. F. BeCKENBACH
B. H. NEUMANN
F. WOLF
K. YoshidA

\section{SUPPORTING INSTITUTIONS}

UNIVERSITY OF BRITISH COLUMBIA

CALIFORNIA INSTITUTE OF TECHNOLOGY

UNIVERSITY OF CALIFORNIA

MONTANA STATE UNIVERSITY

UNIVERSITY OF NEVADA

NEW MEXICO STATE UNIVERSITY

OREGON STATE UNIVERSITY

UNIVERSITY OF OREGON

OSAKA UNIVERSITY

\author{
UNIVERSITY OF SOUTHERN CALIFORNIA \\ STANFORD UNIVERSITY \\ UNIVERSITY OF TOKYO \\ UNIVERSITY OF UTAH \\ WASHINGTON STATE UNIVERSITY \\ UNIVERSITY OF WASHINGTON

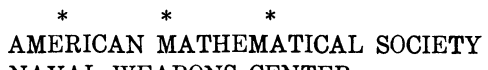 \\ NAVAL WEAPONS CENTER
}

The Supporting Institutions listed above contribute to the cost of publication of this Journal, but they are not owners or publishers and have no responsibility for its content or policies.

Mathematical papers intended for publication in the Pacific Journal of Mathematics should be in typed form or offset-reproduced, (not dittoed), double spaced with large margins. Underline Greek letters in red, German in green, and script in blue. The first paragraph or two must be capable of being used separately as a synopsis of the entire paper. The editorial "we" must not be used in the synopsis, and items of the bibliography should not be cited there unless absolutely necessary, in which case they must be identified by author and Journal, rather than by item number. Manuscripts, in duplicate if possible, may be sent to any one of the four editors. Please classify according to the scheme of Math. Rev. Index to Vol. 39. All other communications to the editors should be addressed to the managing editor, Richard Arens, University of California, Los Angeles, California, 90024.

50 reprints are provided free for each article; additional copies may be obtained at cost in multiples of 50 .

The Pacific Journal of Mathematics is issued monthly as of January 1966. Regular subscription rate: $\$ 48.00$ a year (6 Vols., 12 issues). Special rate: $\$ 24.00$ a year to individual members of supporting institutions.

Subscriptions, orders for back numbers, and changes of address should be sent to Pacific Journal of Mathematics, 103 Highland Boulevard, Berkeley, California, 94708.

PUBLISHED BY PACIFIC JOURNAL OF MATHEMATICS, A NON-PROFIT CORPORATION

Printed at Kokusai Bunken Insatsusha (International Academic Printing Co., Ltd.), 270, 3-chome Totsuka-cho, Shinjuku-ku, Tokyo 160, Japan. 


\section{Pacific Journal of Mathematics}

\section{Vol. 45, No. $2 \quad$ October, 1973}

Kenneth Paul Baclawski and Kenneth Kapp, Induced topologies for quasigroups and loops ............................................. 393

D. G. Bourgin, Fixed point and $\min -\max$ theorems $\ldots \ldots \ldots \ldots \ldots \ldots \ldots$

J. L. Brenner, Zolotarev's theorem on the Legendre symbol ............... 413

Jospeh Atkins Childress, Jr., Restricting isotopies of spheres .............. 415

John Edward Coury, Some results on lacunary Walsh series ................ 419

James B. Derr and N. P. Mukherjee, Generalized Sylow tower groups. II . . . . . . 427

Paul Frazier Duvall, Jr., Peter Fletcher and Robert Allen McCoy, Isotopy Galois

spaces .......................................... 435

Mary Rodriguez Embry, Strictly cyclic operator algebras on a Banach space ... 443

Abi (Abiadbollah) Fattahi, On generalizations of Sylow tower groups ......... 453

Burton I. Fein and Murray M. Schacher, Maximal subfields of tensor products . . 479

Ervin Fried and J. Sichler, Homomorphisms of commutative rings with unit

element .......................................... 485

Kenneth R. Goodearl, Essential products of nonsingular rings ............. 493

George Grätzer, Bjarni Jónsson and H. Lakser, The amalgamation property in

equational classes of modular lattices ...........................

507

$\mathrm{H}$. Groemer, On some mean values associated with a randomly selected simplex

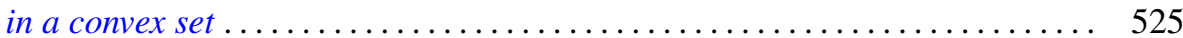

Marcel Herzog, Central 2-Sylow intersections .................... 535

Joel Saul Hillel, On the number of type-k translation-invariant groups ........ 539

Ronald Brian Kirk, A note on the Mackey topology for $\left(C^{b}(X)^{*}, C^{b}(X)\right) \ldots \ldots .543$

J. W. Lea, The peripherality of irreducible elements of lattice.............. 555

John Stewart Locker, Self-adjointness for multi-point differential operators ..... 561

Robert Patrick Martineau, Splitting of group representations ............... 571

Robert Massagli, On a new radical in a topological ring ................. 577

James Murdoch McPherson, Wild arcs in three-space. I. Families of Fox-Artin

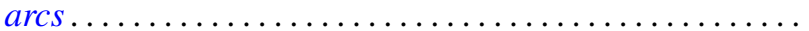

585

James Murdoch McPherson, Wild arcs in three-space. III. An invariant of

oriented local type for exceptional arcs . . . . . . . . . . . . ............ 599

Fred Richman, The constructive theory of countable abelian p-groups ........ 621

Edward Barry Saff and J. L. Walsh, On the convergence of rational functions

which interpolate in the roots of unity ..................

Harold Eugene Schlais, Non-aposyndesis and non-hereditary

decomposability..................................... 643

Mark Lawrence Teply, A class of divisible modules................... 653

Edward Joseph Tully, Jr., H-commutative semigroups in which each

homomorphism is uniquely determined by its kernel ................. 669

Garth William Warner, Jr., Zeta functions on the real general linear group ...... 681

Keith Yale, Cocyles with range $\{ \pm 1\} \ldots \ldots \ldots \ldots \ldots \ldots \ldots \ldots \ldots \ldots \ldots \ldots \ldots . \ldots \ldots$

Chi-Lin Yen, On the rest points of a nonlinear nonexpansive semigroup ........ 699 